\title{
Affinely Adjustable Robust Volt/VAr Control without Centralized Computations
}

This paper was downloaded from TechRxiv (https://www.techrxiv.org).

\section{LICENSE}

CC BY 4.0

SUBMISSION DATE / POSTED DATE

24-08-2021 / 30-08-2021

\section{CITATION}

Nazir, Firdous Ul; Pal, Bikash; Jabr, Rabih (2021): Affinely Adjustable Robust Volt/VAr Control without Centralized Computations. TechRxiv. Preprint. https://doi.org/10.36227/techrxiv.16432329.v1

DOI 


\title{
Affinely Adjustable Robust Volt/VAr Control without Centralized Computations
}

\author{
Firdous U. Nazir, Member, IEEE, Bikash C. Pal, Fellow, IEEE, and Rabih A. Jabr, Fellow, IEEE
}

\begin{abstract}
This paper proposes a completely non-centralized Volt/VAr control (VVC) algorithm for active distribution networks which are faced with voltage magnitude violations due to the high penetration of solar photovoltaics (PVs). The proposed VVC algorithm employs a two-stage architecture where the settings of the classical voltage control devices (VCDs) are decided in the first stage through a distributed optimization engine powered by the alternating direction method of multipliers (ADMM). In contrast, the PV smart inverters are instructed in the second stage through linear $Q(P)$ decision rules - which are computed in a decentralized manner by leveraging robust optimization theory. The key to this non-centralized VVC routine is a proposed network partition methodology (NPM) which uses an electrical distance metric based on node $Q-|V|^{2}$ sensitivities for computing an intermediate reduced graph of the network, which is subsequently divided into the final partitions using the spectral clustering technique. As a result, the final network partitions are connected, stable, close in cardinality, contain at least one PV inverter for zonal reactive power support, and are sufficiently decoupled from each other. Numerical results on the UKGDS-95 bus system show that the non-centralized solutions match closely with the centralized robust VVC schemes, thereby significantly reducing the voltage violations compared to the traditional deterministic VVC routines.
\end{abstract}

Index Terms-Network partitioning, Reactive power decision rules, Robust Optimization, Second order conic relaxation, Volt/VAr control (VVC).

\section{INTRODUCTION}

The Volt/VAr control, as part of the distribution management systems, primarily aims at maintaining an acceptable voltage profile throughout the electricity network. In most cases, the node voltage magnitudes should stay between the upper and lower thresholds of $0.95 \mathrm{pu}$ and $1.05 \mathrm{pu}$. The VVC decides settings for voltage control devices (VCDs) installed on the network through an optimization framework, which requires information about load consumption, PV power generation, network topology, and electrical parameters. The classical VCDs include transformers, on-load tap changers (OLTCs), voltage regulators (VRs), and capacitor banks (CBs), whereas smart PV inverters are also recently being employed in some advanced voltage control routines.

Nowadays, the main challenge the traditional deterministic VVC algorithms face is the uncertainty of the PV real power generation. This is because the VVC solutions are dispatched with a typical periodicity of 15-30 minutes, during which the PV real power generation may exhibit enough variations

F. U. Nazir and B. C. Pal are with the Electrical and Electronic Engineering Department, Imperial College, London SW7 2AZ, U.K. (e-mail: f.ulnazir16@imperial.ac.uk; b.pal@imperial.ac.uk).

R. A. Jabr is with the Department of Electrical and Computer Engineering, American University of Beirut, Beirut 1107 2020, Lebanaon (e-mail: rabih.jabr@aub.edu.lb). to give rise to significant voltage violations [1]. However, simply dispatching the VVC solutions more often is not practically possible owing to the fact that the classical VCDs are switched-type devices which means these are inherently slow in operation, and frequent switching will reduce their life expectancy. In such a situation, reactive power support from PV inverters has been identified as the potential solution by the researchers [2]-[4], given the power electronic nature of the inverters, which enables them to respond almost instantly with negligible wear and tear issues. This fact is also acknowledged by the IEEE 1547-2018 standard, which serves as a specific modification to the original IEEE 1547-2003 standard, to allow dynamic reactive power-based voltage support from the smart inverters [5]. This leads to the problem of the coordinated voltage control between the legacy VCDs and the PV inverters, which has been a subject of recent research. Many research works adopt a control law for adjusting the smart inverter reactive power in response to fluctuations in either the voltage magnitude [6]-[8] or active power [1], [9][12] at their terminals, thereby effectively allowing the legacy VCDs to run at a slower time scale.

In practice, both the parameters of the control law and the settings of the legacy VCDs are computed simultaneously at the beginning of the optimization horizon. The $Q(V)$ control law, adopted in [6] - [8], suffers from the main problem of restrictive convergence conditions and can lead to undesirable oscillatory behaviour, whereas the $Q(P)$ control law has shown stable performance to maintain the voltages in an acceptable region [1], [9]-[12]. Furthermore, the German Grid Code also proposes such $Q(P)$ characteristics to support the network voltage profile [13]. These $Q(P)$ characteristics are mostly decided based on affine policies [1], [11], [12] whereas quadratic decision rules have been used in [9], [10]. Although the quadratic decision rules allow finer control, their use is limited by the tractability of the underlying optimization framework. As an example, the robust optimization framework of [1], [12] only admits a tractable deterministic counterpart when the control law is determined by affine policies [14]. Such affinely adjustable robust optimization framework has superior performance over other techniques like the chance-constrained optimization, used by [10], [11], in that it guarantees that the voltage violations are completely removed provided the reactive power demanded by the optimized control law does not violate the inverter apparent power limits and the network model is linear. However, one of the main limitations for practical implementation of these robust optimization-based frameworks is that their optimization engine is powered by centralized solvers and thus relies heavily on network observability, remote monitoring, and strong communication in- 
frastructure. Moreover, these robust optimization frameworks fail to admit a distributed solution as their decomposability is limited by the presence of many cross-coupling terms, and they require access to the network-wide Newton-Raphson load flow based Jacobian matrix [1]. A recent attempt to achieve distributed solutions for the robust optimization VVC problem was by solving the worst-case realization through a columnand-constraint generation algorithm [15]. However, in this case, the solutions are too conservative because of the absence of second-stage recourse or adjustable variables. This paper proposes a novel algorithm to obtain an affinely adjustable robust optimization solution for the VVC problem without any centralized computations.

The key to our method is identifying neighbourhoods or zones around groups of PVs, such that the node voltage magnitudes within a particular group are much more strongly influenced by the reactive power injections from the PVs of the same zone only. To this end, we propose a novel network partition algorithm based on the spectral clustering technique discussed in the next section. Network partition-based zonal voltage control is a powerful tool and has been around for more than three decades owing to its ease of implementation [16]. Recently, distributed generation sources were grouped for voltage control in a typical microgrid in [17], whereas in [18], a community detection algorithm was used to partition the distribution network for zonal voltage control. Furthermore, the k-means partitioning algorithm was used for steady-state voltage control in a distribution network [19]. In contrast, a power flow tracing and an agglomerative algorithm were proposed in [20] for voltage control of large power systems. A decentralized zonal voltage control realized through multiagent fuzzy logic systems is presented in [21]. However, all these partition-based methods focus on deterministic voltage control strategies. Our proposed NPM, for the first time to the best of our knowledge, attempts to solve the affinely adjustable robust optimization version of the voltage control problem. Furthermore, the final partitions obtained through our proposed NPM are insensitive to the network operating state. This means the partitions are considered permanent until a network reinforcement is needed or a major network reconfiguration event occurs.

To realize the complete voltage control without any type of centralized computations, we employ a two-stage VVC optimization framework, in which the first stage is solved through a distributed solver via the consensus-based ADMM algorithm [22], while the second stage is solved in a decentralized fashion. The distributed solvers achieve solutions as good as the centralized ones with a bit of information exchange at the boundary of the partitions [23]. In this way, optimal deterministic set points of the classical VCDs and the PV smart inverters are decided in the first stage. Assuming that the first stage deterministic reactive power setpoint for a solar PV inverter at bus $j$ is denoted by $\hat{Q}_{j}^{s}$, then the control law for the smart inverter is of the form $Q_{j}^{s}=\hat{Q}_{j}^{s}+\alpha_{j} \Delta P_{j}^{s}$. The slope $\left(\alpha_{j}\right)$ of this affine $\mathrm{Q}(\mathrm{P})$ control law is then decided in the second stage through decentralized optimization solvers employing a robust optimization framework to minimize the
Nomenclature

\begin{tabular}{|c|c|}
\hline $\mathcal{N}(\mathcal{B})$ & Set of nodes (branches) of the network \\
\hline $\mathcal{P}(\mathcal{C})$ & Set of nodes hosting Solar PVs (Capacitors) \\
\hline $\mathcal{N}^{*}\left(\mathcal{P}^{*}\right)$ & Set of nodes (PV nodes) belonging to a particular zone \\
\hline $\mathcal{T}$ & $\begin{array}{l}\text { Set of branches hosting on-load tap changers or voltage } \\
\text { regulators }\end{array}$ \\
\hline $\bar{X}(\underline{X})$ & Upper (lower) limits of a quantity $X$ \\
\hline & Susceptance of the capacitor hosted at the node $j$ \\
\hline$g_{j}\left(b_{j}\right)$ & $\begin{array}{l}\text { Shunt conductance (susceptance) at node } j \text { in the line } \\
\text { equivalent } \pi \text { model }\end{array}$ \\
\hline$l_{i j}$ & $\begin{array}{l}\text { Square of the branch current magnitude flowing on the } \\
\text { branch }(i j)\end{array}$ \\
\hline$P_{j}\left(Q_{j}\right)$ & Net active (reactive) power injection at node $j$ \\
\hline$P_{j}^{l}\left(Q_{j}^{l}\right)$ & Active (reactive) power injection of the load at node $j$ \\
\hline$P_{j}^{s}\left(Q_{j}^{s}\right)$ & $\begin{array}{l}\text { Active (reactive) power injection of the solar PV unit at } \\
\text { node } j\end{array}$ \\
\hline$P_{i j}\left(Q_{i j}\right)$ & $\begin{array}{l}\text { Active (reactive) power flowing on the line }(i j) \text { which } \\
\text { starts from node } i \text { towards node } j\end{array}$ \\
\hline$Q_{j}^{s}\left(Q_{j}^{c}\right)$ & $\begin{array}{l}\text { Reactive power injected by the solar PV inverter } \\
\text { (shunt capacitor) connected at node } j\end{array}$ \\
\hline$r_{i j}\left(x_{i j}\right)$ & Series resistance/ (reactance) of the line $(i j)$ \\
\hline & Apparent power inject from the solar PV unit at node $j$ \\
\hline$t_{i j}$ & Tap setting of the transformer on the branch $(i j)$ \\
\hline$u_{j}\left(u_{(j)}\right)$ & $\begin{array}{l}\text { Square of the node voltage magnitude for the node } j \\
\text { (fictitious node }(j) \text { ) }\end{array}$ \\
\hline
\end{tabular}

voltage magnitude deviation due to the active power variations $\left(\Delta P_{j}^{s}\right)$. To facilitate the decentralized second stage computations, the node $P-Q-|V|$ sensitivities are needed, which are also computed in a distributed fashion by solving secondorder cone programming based load flow routines [24] and subsequently using the perturb and observe based sensitivity concept [25], [26].

The rest of this paper is organised as follows. Section II presents the proposed network partition methodology, a novel electric distance metric is introduced, suitable for zonal voltage control based paradigms for distribution networks. Section III discusses the proposed two-stage voltage control framework for achieving non-centralized robust voltage control settings. Numerical results on the UKGDS-95 bus system are given in Section IV, which shows that the non-centralized solutions are very similar to the centralized ones. Finally, the paper is concluded in Section V.

\section{Network Partition Methodology}

The huge impact of PV reactive power on the nearby nodes than the furthest ones, and the increase in complexity of a centralized control scheme owing to a high penetration level of PVs makes it reasonable to group strongly coupled nodes together for voltage control. Apart from the key fact that the nodes associated together in a cluster are closely related than the remaining nodes of the network, a meaningful NPM for VVC must produce clusters which are a) connected, in the sense that any pair of nodes within a cluster are traceable through a series of edges; b) stable, so that the clusters remain unchanged for different loading and generation patterns of the network; and c) approximately similar size, which implies that the number of nodes in other clusters is not widely different. Furthermore, each cluster must have at least one PV for supplying the leading or lagging VARs required for its voltage control. 


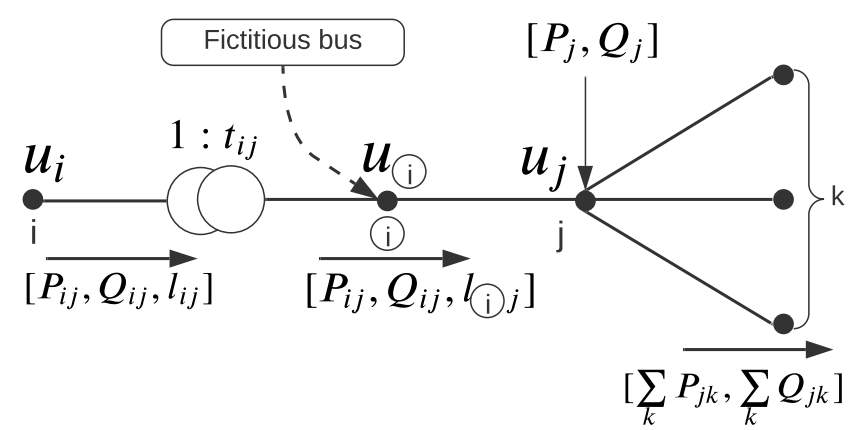

Fig. 1. Typical Radial Distribution Network Section

\section{A. Electrical Distances for Voltage Control}

The concept of electrical distances is used to quantify the proximity between any two nodes of the network. For a voltage control scheme, the electrical distance between two nodes needs to consider a suitable parameter, representing the reactive power to node voltage sensitivity between them. Thus, we use the linearised DisFlow model [27] and extend it to include the transformers present in the network. Consider a radial distribution network, as shown in Fig. 1, the extended linear DistFlow model is given as:

$$
\begin{aligned}
& P_{j}=\sum_{k} P_{j k}-P_{i j} \\
& Q_{j}=\sum_{k} Q_{j k}-Q_{i j} \\
& u_{i}-u_{j}=2\left(r_{i j} P_{i j}+x_{i j} Q_{i j}\right) \\
& u_{(i)}=t_{i j}^{2} u_{i}
\end{aligned}
$$

If there is no transformer present on the branch (ij), then $t_{i j}=$ 1. Thus (1c) and (1d) can be combined as follows:

$$
t_{i j}^{2} u_{i}-u_{j}=2\left(r_{i j} P_{i j}+x_{i j} Q_{i j}\right)
$$

Let $\mathcal{G}(\mathcal{N}, \mathcal{B})$ represent the directed graph of the network with incidence matrix denoted by $\hat{\mathbf{I}}_{n \times(n-1)}$, such that if branch $b$ is sand-witched between nodes $i$ and $j$, then

$$
\begin{aligned}
& \hat{\mathbf{I}}(i, b)=-1 \\
& \hat{\mathbf{I}}(j, b)=1 \\
& \hat{\mathbf{I}}(k, b)=0 \quad \forall k \neq i, j
\end{aligned}
$$

The first row of the incidence matrix denotes the transmission system feed-in point of the network. If we partition the incidence matrix along this row and denote the sub-matrix corresponding to the remaining network as $\mathbf{I}_{(n-1) \times(n-1)}$, then (1a) and (1b) can be written as:

$$
\begin{aligned}
& \mathbf{p}=\mathbf{I} \overrightarrow{\mathbf{p}} \\
& \mathbf{q}=\mathbf{I} \overrightarrow{\mathbf{q}}
\end{aligned}
$$

Also, let us define a matrix $\hat{\mathbf{T}}_{(n-1) \times n}$ from the set of branches $\mathcal{B}$ to the set of nodes $\mathcal{N}$ such that:

$$
\begin{aligned}
& \hat{\mathbf{T}}(b, i)=t_{i j}^{2} \\
& \hat{\mathbf{T}}(b, j)=-1 \\
& \hat{\mathbf{T}}(b, k)=0 \quad \forall k \neq i, j
\end{aligned}
$$

Thus we can write (2) as follows:

$$
\hat{\mathbf{T}} \hat{\mathbf{u}}=2 \operatorname{diag}(\mathbf{r}) \overrightarrow{\mathbf{p}}+2 \operatorname{diag}(\mathbf{x}) \overrightarrow{\mathbf{q}}
$$

The first column of $\hat{\mathbf{T}}$ corresponds to the transmission system feed in point. Denote this column vector by $\mathbf{t}_{1}$ and the rest of the matrix as $\mathbf{T}$. Then (4) is written as:

$$
\left[\begin{array}{ll}
\mathbf{t}_{1} & \mathbf{T}
\end{array}\right]\left[\begin{array}{c}
u_{1} \\
\mathbf{u}
\end{array}\right]=2 \operatorname{diag}(\mathbf{r}) \overrightarrow{\mathbf{p}}+2 \operatorname{diag}(\mathbf{x}) \overrightarrow{\mathbf{q}}
$$

Thus the squared node voltage magnitudes for all but the substation node can be obtained by the closed-form solution as follows:

$$
\mathbf{u}=2 \mathbf{T}^{-1} \operatorname{diag}(\mathbf{r}) \mathbf{I}^{-1} \mathbf{p}+2 \mathbf{T}^{-1} \operatorname{diag}(\mathbf{x}) \mathbf{I}^{-1} \mathbf{q}-\mathbf{T}^{-1} \mathbf{t}_{1} u_{1}
$$

If voltage control is done through reactive power modulation, assume that the vector $\delta \mathbf{q}$ gives the change in nodal reactive power injections, then the corresponding change in squared node voltage magnitudes is given by:

$$
\delta \mathbf{u}=\underbrace{2 \mathbf{T}^{-1} \operatorname{diag}(\mathbf{x}) \mathbf{I}^{-1}}_{\mathbf{\Lambda}} \delta \mathbf{q}
$$

The matrix $\Lambda$ represents a matrix of $Q-|V|^{2}$ sensitivities. For easy analysis, $\boldsymbol{\Lambda}$ is normalized so that the diagonal entries are unity, thereby revealing the strength of cross-sensitivities or off-diagonal entries. Let the normalized matrix be denoted by $\Xi$, such that

$$
\Lambda=\Gamma \Xi
$$

Where, $\boldsymbol{\Gamma}=\operatorname{diag}(\boldsymbol{\Lambda})$, is positive definite and invertible for a network with stable voltages, meaning that voltage collapse condition does not exist. Since $\boldsymbol{\Gamma}$ is invertible, we can combine (7) and (8) in the following manner:

$$
\underbrace{\Gamma^{-1} \delta \mathbf{u}}_{\delta \mathbf{u}^{*}}=\boldsymbol{\Xi} \delta \mathbf{q}
$$

The vector $\delta \mathbf{u}^{*}$ represents a scaled squared voltage magnitude deviation vector. Each entry represents the corresponding squared node voltage deviation being scaled down by the self $Q-|V|^{2}$ sensitivity of that node. Since these scaled deviations are linked to the reactive power modulations through $\boldsymbol{\Xi}$, we define the electrical distances through the operation given below:

$$
\mathcal{D}=-\ln \left(\boldsymbol{\Xi} \circ \boldsymbol{\Xi}^{T}\right)
$$

o denotes the Hadamard (element-by-element) product. The matrix $\mathcal{D}$ is always hollow, symmetric, and nonnegative. Furthermore, for a network that is not over-compensated, in the sense that the inductive terms are not eliminated but only attenuated by the capacitive devices, the triangular inequality for any three nodes with respect to $\mathcal{D}$ will also hold [16]. Thus matrix $\mathcal{D}$ represents a true distance matrix. It is important to note that these electrical distances do not change with 
generation and loading patterns. Furthermore, our simulation studies reveal that the effect of transformer/OLTC taps as captured by the matrix $\mathbf{T}$ is negligible for practical load curves. Thus, using distance matrix $\mathcal{D}$ for carrying out the network partitioning results in stable clusters.

\section{B. Reduced Graph}

The distance matrix $\mathcal{D}$ can assign each node of the network to its nearest PV node. This assignment exercise, therefore, identifies the nearest neighbours around each PV node. Denoting each such neighbourhood by a fictional node and later connecting them through branches that correspond to actual branches on the network results in a much smaller graph known as the reduced graph. More specifically, two fictional nodes in the reduced graph would be connected if there is a branch between any pair of nodes in the original graph, which has been assigned to these fictional nodes. In this way, the number of nodes in the reduced graph is equal to the number of PV buses. For the assignment exercise, we initially define an orthonormal standard basis matrix, $\Psi \in \mathbb{R}^{m \times n-1}$, with each row representing a basis vector of the vector space in $\mathbb{R}^{n-1}$. Here $m$ represents the number of PVs and $n$ represents the number of nodes in the network. Thus each row of $\Psi$ corresponds to a particular PV in the network and will have exactly one non-zero entry whose value is 1 with indices decided by the location of the specific PV in the network. Let us say that the $i^{\text {th }} \mathrm{PV}$ is connected at bus $j$ in the network, then:

$$
\boldsymbol{\Psi}(i, k)=1: i \leq m, k=j-1, j \leq n
$$

The basis matrix $\Psi$ is used to extract a reduced distance matrix, denoted by $\mathcal{D}_{r}$, whose columns correspond to the PV nodes, as follows:

$$
\mathcal{D}_{r}=\boldsymbol{\Psi} \mathcal{D}
$$

We use the proposed reduced distance matrix $\mathcal{D}_{r}$ to complete the assignment exercise by computing the linear index of the least value entry in each row. The vector of such indices, denoted by $\theta$, therefore represents a direct mapping of the original network nodes to the reduced graph and is mathematically computed as:

$$
\theta_{i}=\underset{j}{\arg \min } \mathcal{D}_{r}(i, j), \quad i=1, \ldots, n-1
$$

It is evident from (13) that the value of a particular entry directly gives the fictitious node of the reduced graph to which the corresponding node of the original network belongs. In this way, $m$ connected clusters are identified, which represent nodes of the reduced graph.

\section{Spectral Clustering on the Reduced Graph}

The final clustering of the original network is achieved by performing spectral clustering on the reduced graph. The essence of spectral clustering is to map the network nodes into a low dimensional space, characterized by the lower eigenvalues of the graph Laplacian matrix $\mathbf{L}$, that can be easily segregated into clusters. We build the Laplacian by

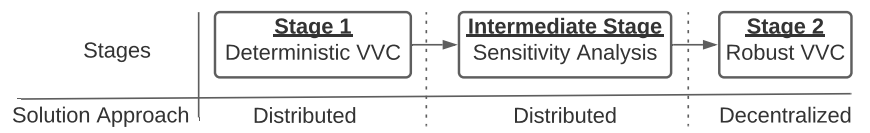

Fig. 2. Two Stage VVC Implementation

initially computing the graph similarity matrix, $\mathbf{W}$, for which we propose the following procedure:

- Associate each node of the reduced graph with a weight equal to the cardinality of the subnetwork represented by that fictional node. Let $\mathbf{W}_{i}$ be the weight of the node $i$.

- Construct similarity matrix $\mathbf{W}$, as given:

$$
\mathbf{W}_{i j}= \begin{cases}\frac{1}{\mathbf{W}_{i}}+\frac{1}{\mathbf{W}_{j}} & \text { if nodes } i \text { and } j \text { are connected } \\ 0 & \text { Otherwise }\end{cases}
$$

The proposed similarity matrix is a modified adjacency matrix of the reduced graph. Furthermore, it gives lower weights to the edges connecting the nodes that represent bigger subnetworks of the original graph. These edge weights measure the connection strength between two nodes, as weakly connected nodes are more likely to separate when clustering. The procedure ensures that the final clusters have a relatively equal number of nodes. Thus, there is an established power engineering interpretation of the proposed similarity matrix. Finally the Laplacian matrix is computed with the help of the proposed similarity matrix as:

$$
\mathbf{L}=\mathbf{D}-\mathbf{W}
$$

Here, $\mathbf{D}$ is the degree matrix, a diagonal matrix with entries equal to the sum of the corresponding row of the similarity matrix [28]. Thus, degree matrix D : $\mathbf{D}_{i i}=\sum_{j} \mathbf{W}_{i j}$. Given the Laplacian matrix $\mathbf{L}$ and the number of clusters needed, we partition the network by employing spectral clustering methodology [29] on the reduced graph. The final partitions reveal the different clusters of the original network, and the cut edges along which the partitions are made.

\section{NON-CENTRALIZED Volt/VAR CONTROL}

Once the distribution network has been partitioned into optimal zones by the NPM as described above, the voltage control of the network is achieved without the need for a centralized controller. Instead, each revealed zone is controlled by its local controller. The settings for the proposed noncentralized robust voltage control are achieved by solving two separate VVC frameworks in sequence together with an intermediate sensitivity analysis routine, as shown in Fig. 2. The three important blocks in Fig. 2 are discussed in detail below.

\section{A. Stage 1: Deterministic VVC}

The deterministic VVC seeks to minimize the total active power loss of the network by optimizing settings for the classical VCDs and the deterministic reactive powers for PV inverters based on the forecast values of the PV active power generations. The voltage magnitudes are held within the VVC 
thresholds using strict box constraints, while the load flow model of the system is convexified through second-order cone relaxations. These second-order cone relaxations are guaranteed to be exact under milder and practical assumptions for radial distribution networks [30]. Thus, in the first stage, the following second-order cone program is solved.

$$
\begin{aligned}
& \min \sum_{(i j) \in \mathcal{B} / \mathcal{T}} r_{i j} l_{i j}+\sum_{(i j) \in \mathcal{T}} r_{i j} l_{(i)} \\
& \text { s.t. } P_{j}^{l}+P_{j}^{s}=\sum_{k: j \rightarrow k} P_{j k}-\sum_{i: i \rightarrow j}\left(P_{i j}-r_{i j} l_{i j}\right)+g_{j} u_{j} \text {, } \\
& \forall j \in \mathcal{N} \\
& Q_{j}^{l}+Q_{j}^{c}+\widehat{Q_{j}^{s}}+b_{j} u_{j}=\sum_{k: j \rightarrow k} Q_{j k} \\
& -\sum_{i: i \rightarrow j}\left(Q_{i j}-x_{i j} l_{i j}\right) \\
& \forall j \in \mathcal{N} \\
& u_{j}=u_{i}-2\left(r_{i j} P_{i j}+x_{i j} Q_{i j}\right)+\left(r_{i j}^{2}+x_{i j}^{2}\right) l_{i j}, \\
& \forall(i j) \in \mathcal{B} / \mathcal{T} \\
& u_{j}=u_{(i}-2\left(r_{i j} P_{i j}+x_{i j} Q_{i j}\right)+\left(r_{i j}^{2}+x_{i j}^{2}\right) l_{(i) j}, \\
& \forall(i j) \in \mathcal{T} \\
& \left\|\begin{array}{c}
2 P_{i j} \\
2 Q_{i j} \\
l_{i j}-u_{i}
\end{array}\right\|_{2} \leq l_{i j}+u_{i} \\
& \forall(i j) \in \mathcal{B} \\
& \left\|\begin{array}{c}
2 P_{i j} \\
2 Q_{i j} \\
l_{(i) j}-u_{(i)}
\end{array}\right\|_{2} \leq l_{(i) j}+u_{(i)}, \quad \forall(i j) \in \mathcal{T} \\
& t_{i j}^{2} u_{i} \leq u_{(i)} \leq \overline{t_{i j}^{2}} u_{i} \text {, } \\
& \forall i \in \mathcal{T} \\
& b_{j}^{c} u_{j} \leq Q_{j}^{c} \leq \overline{b_{j}^{c}} u_{j}, \\
& \forall j \in \mathcal{C} \\
& -\sqrt{\left(\overline{S_{j}^{s}}\right)^{2}-\left(P_{j}^{s}\right)} \leq \widehat{Q_{j}^{s}} \leq \sqrt{\left(\overline{S_{j}^{s}}\right)^{2}-\left(P_{j}^{s}\right)}, \\
& \forall j \in \mathcal{P} \\
& l_{i j} \leq \overline{l_{i j}} \text {, } \\
& \forall(i j) \in \mathcal{B} \\
& \underline{u_{j}} \leq u_{j} \leq \overline{u_{j}} \text {, } \\
& \forall j \in \mathcal{N} / 1 \\
& u_{0}=1 \text {, } \\
& \text { Substation bus }
\end{aligned}
$$

$(16 \mathrm{~m})$

The objective of the above optimization problem, as defined in (16a), minimizes the branch active power losses over the entire network. The feasible convex region for this optimization problem is demarcated by the constraints defined through (16b) - (16m). The real power and reactive power balance of a particular node are respectively denoted by (16b) and (16c); it is obvious that these power balances are determined by the type of devices connected at that node. Also, if the incoming branch hosts a transformer, the appropriate value of $l_{i j}$, which is $l_{(i)}$, is used in these equations. The Ohms law governing the voltage drop in the branches without and with a transformer is represented by (16d) and (16e). The actual load flow model needs the branch flow quantities to follow the nonconvex quadratic relation given by $P_{i j}^{2}+Q_{i j}^{2}=l_{i j} u_{i}$, which is relaxed to an exact second-order conic (SOC) constraints through (16f). Furthermore, it is clear that (16g) enforces additional SOC constraints on branches that host transformers. However, this extra cone features the same active $\left(P_{i j}\right)$ and reactive power flows $\left(Q_{i j}\right)$, implying that the transformers are considered lossless. Finally, (16h) - (16m) hold various optimization variables within their permissible bounds. Note that continuous relaxations are used through (16h) and (16i) to retain the model's convexity. (16j) limits the reactive power supplied by a PV inverter inside its PQ capability curve as dictated by the maximum apparent power capacity and the forecasted real power.

The deterministic optimization framework set up in (16) is separable with respect to any splitting in the objective function as all the constraints feature only immediately local variables and parameters. This is to say that a constraint defined for the network nodes, e.g. (16b), only depends on quantities related to either the devices or branches connected to the particular node. Similarly, a constraint defined for a particular branch, e.g. (16d), only depends on quantities related to that branch and the corresponding end nodes. Thus we can write separate deterministic VVC subproblems for all the partitions obtained by the NPM in the previous section. In order to achieve a global optimum solution for the framework of (16), we use a consensus-based ADMM algorithm to solve these subproblems in a distributed fashion [9]. However, the ADMM algorithm requires the adjacent zones to overlap to allow more than one local controller to compute the decision variables belonging to these overlapping regions. Subsequently, the ADMM algorithm pushes the different local controllers to agree on the values for such decision variables. Once this consensus is achieved, the algorithm stops and the solutions are mathematically guaranteed to be the same as those of a centralized solver, given that the optimization framework is convex [23]. We effectively use the cut edges or branches, along which the NPM partitions the network into various zones, for providing the ADMM with required overlap regions. In this work, all the adjacent zones share any such cut branches along and end nodes to achieve the required consensus. Once the deterministic VVC converges, the optimum schedule of the classical VCDs is available in an implicit way. For a transformer hosted in the branch $(i j)$ the tap ratio is obtained from $\sqrt{\frac{u^{(i)}}{u_{i}}}$ after rounding off to the nearest discrete value. Similarly, the capacitor susceptance value at node $j$ is given by $\frac{Q_{j}^{c}}{u_{j}}$. Moreover, the deterministic value of the PV reactive power is directly available from the optimizer.

\section{B. Distributed Sensitivity Analysis Approach}

Once the dispatches are available from the deterministic VVC routine, the sensitivity coefficients $(P-Q-|V|)$ for network nodes corresponding to this operating state need to be computed for use in the second stage robust VVC. Mainly these sensitivities are obtained through the inversion of the Jacobian matrix available from Newton-Raphson load flow routine [31]. However, this method can not be used without a centralized computing agent. Thus we adopt the perturb and observe $(\mathrm{P} \& \mathrm{O})$ based sensitivity analysis method [25] and show that it can obtain the node sensitivities in a distributed manner. In the $\mathrm{P} \& \mathrm{O}$ method, the sensitivity coefficients are approximated by injecting a small amount of 
additional active/reactive power at a node and measuring the impact on the network voltage magnitude with respect to the actual operating state. Assuming that the change in voltage magnitude for node $i$ is $\Delta\left|V_{i}\right|$ when the active (reactive) power injection at node $j$ changes by $\Delta P_{j}\left(\Delta Q_{j}\right)$, then the $P-|V|$ $(Q-|V|)$ sensitivity of node $i$ with respect to node $j$ is given as:

$$
\begin{aligned}
K_{i j}^{P|V|} & =\frac{\Delta\left|V_{i}\right|}{\Delta P_{j}} \\
K_{i j}^{Q|V|} & =\frac{\Delta\left|V_{i}\right|}{\Delta Q_{j}}
\end{aligned}
$$

In this work, an injection equalling $1 \%$ of the connected P/Q load value has been used. In addition, an injection equal to $1 \%$ of the smallest non-zero P/Q load value was used for the buses with zero connected load. This process is repeated for all nodes of the network. Thus, this method requires solving $2 n+1$ load flows, when $n$ is the number of nodes in the network. To solve the load flow in a distributed manner, [24] has proved that a SOCP, which is very similar to the framework in (16) albeit much simpler, needs to be solved. The load flow optimizer uses fixed values for the VCDs given by the dispatches from the deterministic VVC in stage 1. Specifically, the lower and upper bounds in (16h) - (16j) become equal as determined by these dispatches. Further, constraints on the node voltage and branch current magnitudes as defined in (16k) - (16l) are not needed.

\section{Stage 2: Decentralized Robust VVC}

The main advantage of using the sensitivity analysis is that it allows linearly quantifying the change in voltage magnitude at a node when the PV real power fluctuates. Assume that the PV real power at bus $j$ fluctuates by $\Delta P_{j}^{s}$ in response of which the second stage robust VVC routine adjusts the corresponding reactive power by $\Delta Q_{j}^{s}=\alpha_{j} \Delta P_{j}^{s}$, then the deviation in voltage magnitude at bus $i$ is given by:

$$
\Delta\left|V_{i}\right|=\sum_{j \in P}\left(K_{i j}^{P|V|}+K_{i j}^{Q|V|} \alpha_{j}\right) \Delta P_{j}^{s}
$$

The second stage of control strives to minimize these voltage deviations due to the changing PV active power injection by optimally adjusting the reactive power feed locally. This is mathematically denoted as:

$$
\begin{array}{cl}
\min \sum_{i \in \mathcal{N}^{*}} \sum_{j \in P^{*}}\left(K_{i j}^{P|V|}+K_{i j}^{Q|V|} \alpha_{j}\right) \Delta P_{j}^{s} & \\
\text { s.t. } Q_{j}^{s}=\hat{Q}_{j}^{s}+\alpha_{j} \Delta P_{j}^{s} & \forall j \in \mathcal{P}^{*} \\
P_{j}^{s}=\hat{P}_{j}^{s}+\Delta P_{j}^{s} & \forall j \in \mathcal{P}^{*} \\
\Delta P_{j}^{s} \in\left[\underline{\Delta P_{j}^{s}}, \overline{\Delta P_{j}^{s}}\right] & \forall j \in \mathcal{P}^{*}
\end{array}
$$

It is important to note that the above optimization framework is set up separately for each zone which is identified by the NPM. This implies that the effect of the reactive power modulation for a PV from one zone is negligible to the node voltage magnitudes of all other zones. This assumption is in line with the basic premise of the NPM. (20d) shows that the PV real power deviation varies in an uncertainty

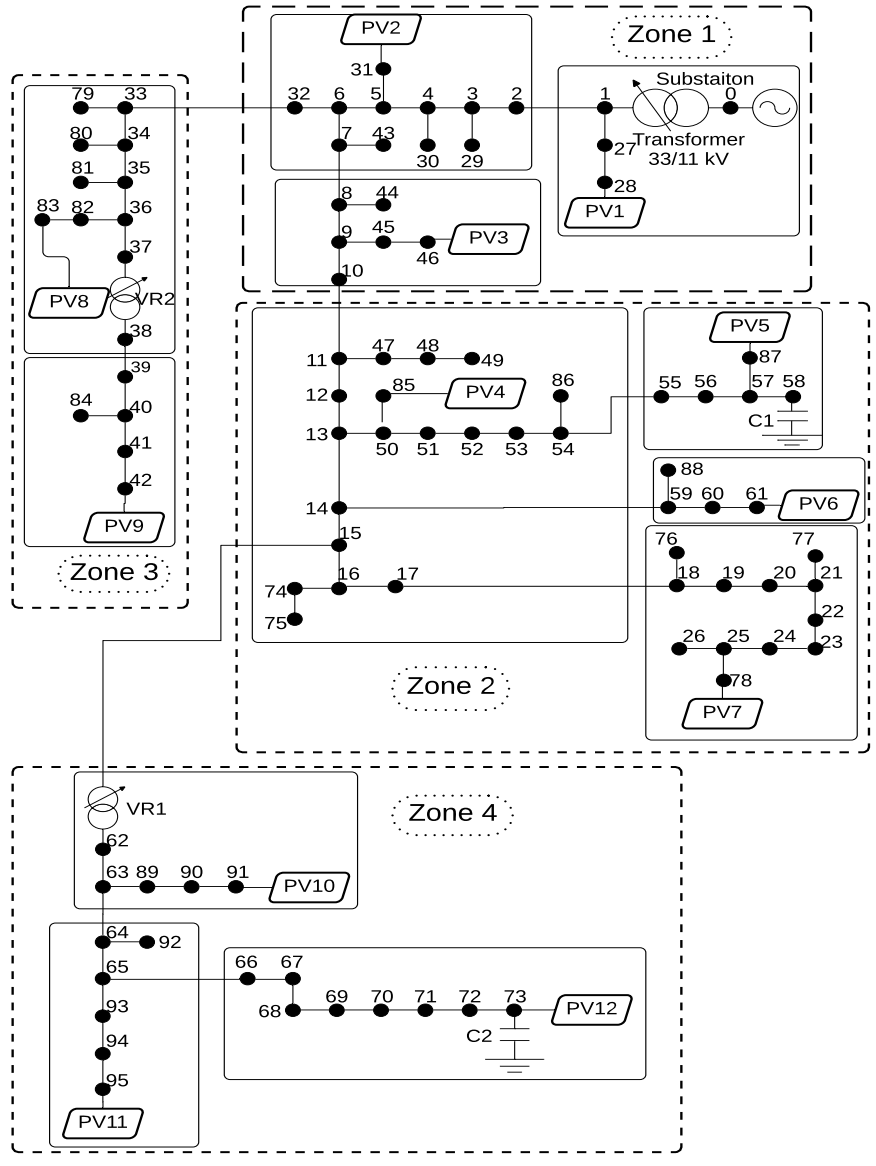

Fig. 3. UKGDS-95 bus system

interval with known upper and lower bounds. This allows us to write the affinely adjustable robust counterpart of the above optimization framework by employing the concept of epigraph programming and using positive/negative slack variables [1], [32] as follows:

$$
\begin{aligned}
& \min \sum_{i \in \mathcal{N}^{*}} t_{i} \\
& \text { s.t. } \sum_{j \in \mathcal{S}^{*}}\left[z_{j i}^{+} \overline{\Delta P_{j}^{g}}+z_{j i}^{-} \underline{\Delta P_{j}^{g}}\right] \leq t_{i}, \quad \forall i \in \mathcal{N}^{*} \\
& \sum_{j \in \mathcal{S}^{*}}\left[z_{j i}^{+}\left(-\underline{\Delta P_{j}^{g}}\right)+z_{j i}^{-}\left(-\overline{\Delta P_{j}^{g}}\right)\right] \leq t_{i}, \quad \forall i \in \mathcal{N}^{*} \\
& z_{j i}^{+} \geq 0 \text {, } \\
& \forall i \in \mathcal{N}^{*}, j \in \mathcal{P}^{*} \\
& z_{j i}^{+} \geq K_{i j}^{P|V|}+K_{i j}^{Q|V|} \alpha_{j}, \quad \forall i \in \mathcal{N}^{*}, j \in \mathcal{P}^{*} \\
& z_{j i}^{-} \leq 0 \text {, } \\
& z_{j i}^{-} \leq K_{i j}^{P|V|}+K_{i j}^{Q|V|} \alpha_{j}, \quad \forall i \in \mathcal{N}^{*}, j \in \mathcal{P}^{*}
\end{aligned}
$$

The above problem is a linear programming problem that can be efficiently solved to compute the optimal value of the slope $\left(\alpha_{j}\right)$ for the PV inverter's affine control law.

\section{Numerical Results}

The numerical computations have been performed on a 3.5 $\mathrm{GHz}$ Intel Xeon E5 processor with 64 GB of RAM, where 


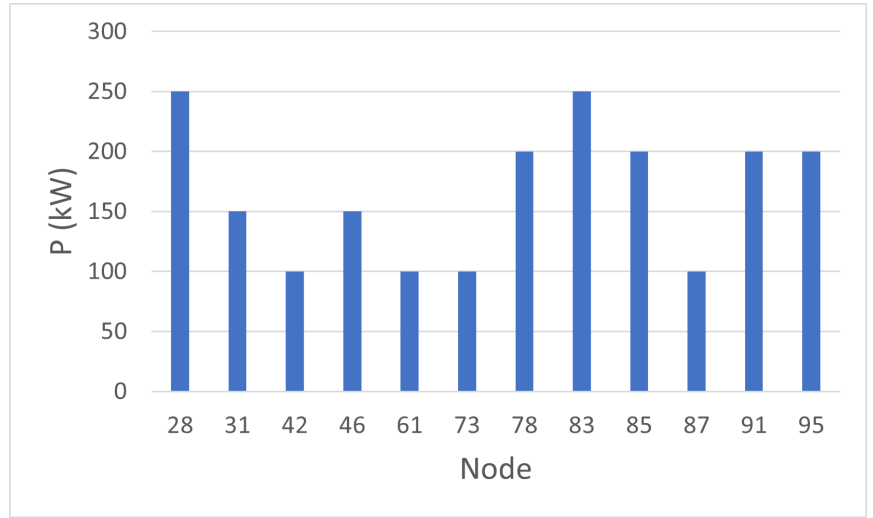

Fig. 4. Generation capacity forecast for the PVs

the proposed NPM was implemented in MATLAB, and the proposed non-centralized VVC framework was programmed in Julia with Gurobi 9.1 [33] employed as the optimizer. The algorithm was tested on the UKGDS-95 bus system shown in Fig. 3, fed by a 33/11 kV substation OLTC transformer. The base voltage of $11 \mathrm{kV}$ and a base power of 10 MVA are used. The network has two VRs - VR1 between buses 15-62 and VR2 between buses 37-38. The OLTC and the VRs have an operating range of 0.9 to 1.1 , spanned by 33 equal steps. There are also two capacitor banks - $\mathrm{C} 1$ hosted at bus 58 and $\mathrm{C} 2$ at bus 73 . Both $\mathrm{C} 1$ and $\mathrm{C} 2$ can supply a maximum of 300 kvar, each having a step size of 50 kvar. The network has 12 solar PV units installed at various nodes, whose generation forecast for the scenario under study is shown in Fig 4. This forecast represents a PV penetration level of $44.16 \%$ of the forecasted network active power load. For each PV unit, the lower bound of its active power uncertain interval, as given by (20d), is taken as -0.4 times the corresponding forecasted value, whereas the upper bound of this interval is set at 0.4 times the forecast. This effectively means that the maximum and minimum generation levels for the PV are $61.82 \%$ and $26.49 \%$ of the load forecast. Moreover, the apparent power capacity of the PV inverters is assumed to be $10 \%$ above the connected active power capacity [4].

\section{A. Performance of the partitioning algorithm}

The proposed partitioning algorithm reveals four zones for the UKGDS-95 bus network. After calculating the reduced distance matrix of the network via (12) and subsequently applying the mapping suggested by (13), twelve clusters are obtained. These intermediate clusters are shown by solid boxes in Fig. (3) and contain precisely one PV node. These clusters define the reduced graph of the network, where each cluster represents the fictional node of the reduced graph. Given the number of nodes in each cluster, the $12 \times 12$ similarity matrix is computed using (14). Finally, the whole network is divided into four zones, demarcated by the dashed line boxes in Fig. 3 . It is clear that the obtained zones are connected. Furthermore, to check the stability of the partitions with respect to changing the operating state of the network, the NPM is applied for an entire year with half-hourly data resolution. To this end,

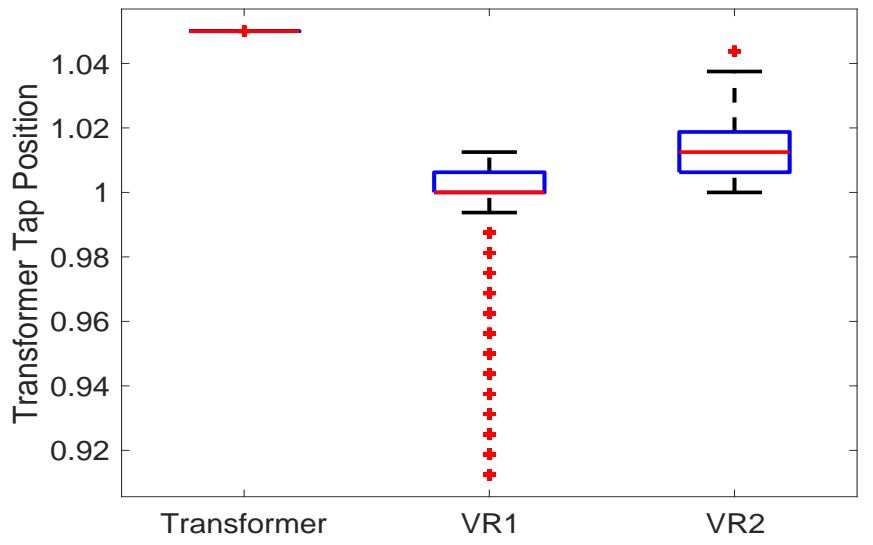

Fig. 5. Transformer taps for an entire year

TABLE I

NUMBER OF NODES IN EACH ZONE

\begin{tabular}{|c||c|c|c|c|}
\hline Zone & 1 & 2 & 3 & 4 \\
\hline Number of Nodes & 20 & 40 & 16 & 19 \\
\hline
\end{tabular}

optimum values of the OLTC and VRs for the selected year are obtained through a deterministic VVC routine. The halfhourly data for the UKGDS-95 bus network is available from [34]. Fig. 5 shows these optimum tap positions, indicating that the OLTC and VR taps have a small variance around their median values. Based on these tap values, the proposed network partition methodology revealed the exact same zones for all the operating states. Moreover, Table I shows that the number of nodes in zones 1, 3, and 4 is comparable. However, zone 2 is much bigger. This is because the location of the PVs allows the intermediate clusters around PV6 and PV7 to coalesce with either the best possible zone (zone 2) or zone 4 only. In either case one of the final zones is going to be bigger than the other three. Thus the proposed partitioning methodology can identify zones that, in most cases, are equal in size.

\section{B. Performance of the non-centralized Volt/VAr control}

To test the comparative performance of the non-centralized voltage control methodology, the UKGDS-95 bus network is operated under the following three voltage control schemes:

- Base Case represents the traditional single-stage VVC, where the PV inverters do not engage in voltage ancillary services.

- Cent AARO represents the centralized affinely adjustable robust optimization-based VVC [1].

- Ncent AARO denotes the proposed non-centralized counterpart.

In the first set of voltage control performance-related tests, the PV inverter slopes $(\alpha)$ for the control law were computed through the 'Cent AARO' [1] and our proposed 'Ncent AARO' as discussed in (21). For quantification purposes, we introduce a metric known as slope deviation factor, denoted 


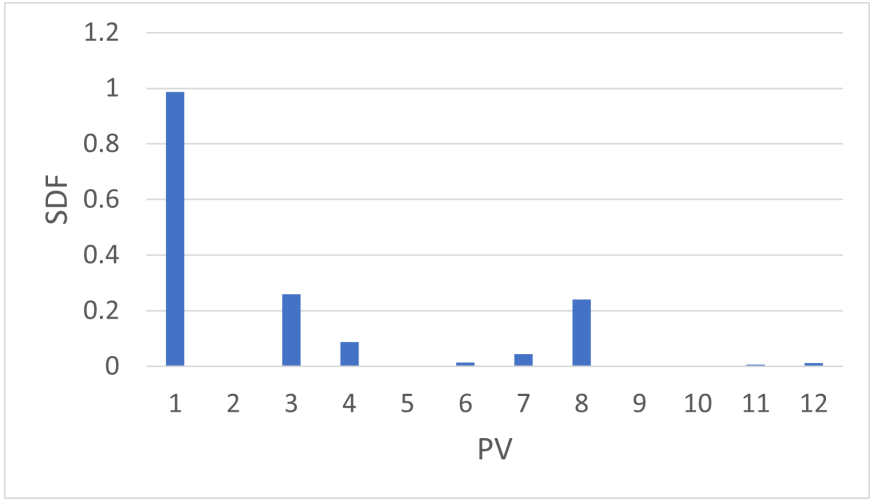

Fig. 6. Slope deviation factor

TABLE II

Percentage nOde Voltage Violations

\begin{tabular}{|c||c|c|}
\hline & PV at max & MC trials average \\
\hline Base Case & 69.79 & 5.15 \\
Cent AARO & 1.04 & 0.14 \\
Ncent AARO & 3.12 & 0.14 \\
\hline
\end{tabular}

by $S D F$, and is defined as:

$$
S D F_{j}=\left|\frac{\alpha_{j}^{c}-\alpha_{j}^{d}}{\alpha_{j}^{c}}\right|
$$

Here, $\alpha_{j}^{c}$ and $\alpha_{j}^{d}$ respectively give the value of the control law slope computed through the 'Cent AARO' and 'Ncent AARO' VVC routines for the $j^{\text {th }}$ inverter, and $S D F_{j}$ is the value of the slope deviation factor for this inverter. The lesser the value of $S D F$, the better agreement between the centralized and decentralized dispatches - and a value of zero implies complete agreement. The slope deviation factors for all the PVs are shown in Fig. 6, and it is clear from this figure that the robust decentralized dispatches are very close to the centralized ones with the same values for four inverters.

Table II shows the percentage of the nodes whose voltage magnitudes violate either the upper $(1.05 \mathrm{pu})$ or the lower $(0.95 \mathrm{pu})$ thresholds when the network is operated under the three different voltage control schemes. One set of tests is performed when all the PVs are allowed to generate the maximum active power determined by the upper bound of the uncertain interval $\left(\overline{\Delta P_{j}^{s}}\right)$, while another set of tests is carried on 500 randomly generated Monte-Carlo trials from the uncertainty interval of the $\mathrm{PV}$ active powers $\left(\left[\Delta P_{j}^{s}, \overline{\Delta P_{j}^{s}}\right]\right)$. Node voltage magnitudes in all the scenarios are obtained through the current injection-based load flow technique [35]. It is clear from Table II that the base case VVC has appreciable voltage violations while the robust optimization-based VVC manages to reduce these voltage magnitude violations significantly. Also, the non-centralized robust VVC can perform almost as effectively as the centralized one. Note that the small voltage violation values in the robust VVC cases result from PV inverters not being able to supply the requested reactive power because the maximum inverter apparent power limits $\left(\overline{S^{s}}\right)$ become active. In such cases, the inverter limits the reactive power at the boundary of its PQ capability curve [1].

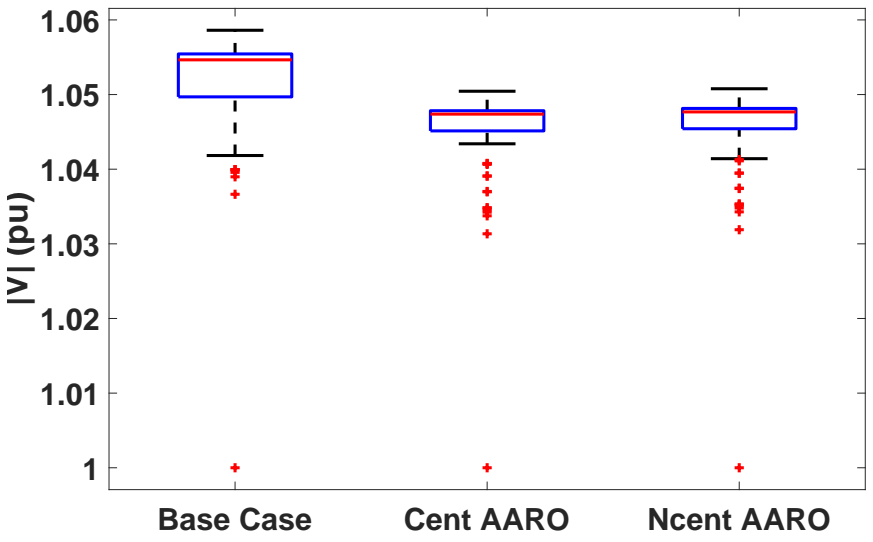

Fig. 7. Comparison of voltage violations for the maximum PV generation scenario for different VVC routines

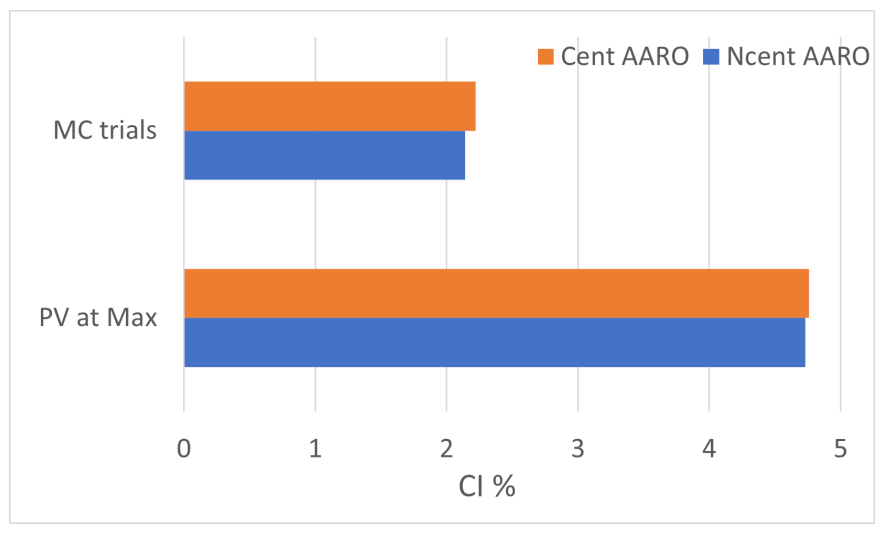

Fig. 8. Conservative index of the robust VVC schemes

Fig. 7 shows the voltage profile of the network under the considered voltage control schemes for the scenario of maximum PV real power generation. The traditional VVC scheme represented as 'Base Case' has many nodes with severe voltage violations. The most severely violated node reaches a voltage magnitude close to $1.06 \mathrm{pu}$, with even the median of the voltage profile around $1.055 \mathrm{pu}$. It is clear from this figure that both the robust optimization routines (Cent AARO and Ncent AARO) effectively push the voltage profile to stay within the permissible limits, with the most severe violations slightly above $1.05 \mathrm{pu}$.

The secondary aim of the network VVC is active power loss minimization. However, the tradeoff for achieving a robust solution is a slight increase in such losses. Fig. 8 shows the conservative index metric, defined as the per unit increase in the active power loss for the robust optimization framework compared with the traditional 'Base Case' VVC. The percentage conservative index is around $5.75 \%$ when the PV operates at maximum active power generation capacity, while an average percentage increase for MC trials is around 2.2\%. Further, the non-centralized robust VVC framework performs slightly better than the centralized robust optimization scheme when it comes to increase in the active power loss. This is expected as the centralized optimization scheme is somewhat 
TABLE III

COMPUTING TIME OF THE CODES

\begin{tabular}{|c||c|c|c|}
\hline \multicolumn{1}{|c||}{ Partitioning } & \multicolumn{3}{|c|}{ Voltage control } \\
\hline \multirow{2}{*}{$26.58(\mathrm{~ms})$} & Sage 1: VVC & Sensitivity analysis & Stage 2: VVC \\
\cline { 2 - 4 } & $4.89(\mathrm{~s})$ & $98.46(\mathrm{~s})$ & $0.59(\mathrm{~s})$ \\
\hline Total & \multicolumn{3}{|c}{$103.94(\mathrm{~s})$} \\
\hline
\end{tabular}

more robust than the non-centralized one.

Finally, the computational overhead required for the partitioning algorithm of Section II and the voltage control scheme of Section III is given in Table III. The Stage 1 VVC and the sensitivity analysis routines use the ADMM algorithm, which is an iterative process and thus inherently slow. However, the reported times are on a serial implementation of the algorithm. In practice the ADMM is implemented through parallel processors, which means that the actual time needed will be decided by the slowest of these processors; a rough estimate can be put at a quarter of the reported times for these ADMM based frameworks owing to four partitions of the network. Also, note that 191 load flow solutions need to be calculated for the sensitivity analysis routine compared to only one solution of the Stage 1 VVC, which implies that each run of the sensitivity analysis algorithm is approximately ten times as fast as the Stage 1 VVC algorithm.

\section{CONCLUSION}

This paper presented a completely non-centralized approach for achieving affinely adjustable robust voltage control decisions on distribution networks with significant PV penetration. The proposed voltage control algorithm is implemented in two stages: the first stage solves a deterministic VVC framework in a distributed manner, while the second stage uses a decentralized approach to solve a robust VVC optimization program. For effective voltage control, the network is divided into various zones through a proposed network partition methodology based on node $Q-|V|$ sensitivities. Owing to this zonal voltage control strategy, the communication overhead is reduced. At the same time, the data privacy of the different utilities, which are in charge of various parts of the network, is guaranteed. The results on the UKGDS-95 bus network show that the voltage violations due to fluctuating PV active powers are significantly reduced when the proposed voltage control is used. Furthermore, these non-centralized decisions match closely to those of the centralized solvers.

\section{REFERENCES}

[1] R. A. Jabr, "Robust volt/var control with photovoltaics," IEEE Transactions on Power Systems, vol. 34, no. 3, pp. 2401-2408, 2019.

[2] F. Olivier, P. Aristidou, D. Ernst, and T. Van Cutsem, "Active management of low-voltage networks for mitigating overvoltages due to photovoltaic units," IEEE Transactions on Smart Grid, vol. 7, no. 2, pp. 926-936, 2016.

[3] M. Bollen and A. Sannino, "Voltage control with inverter-based distributed generation," IEEE Transactions on Power Delivery, vol. 20, no. 1, pp. 519-520, 2005.

[4] K. Turitsyn, P. Sulc, S. Backhaus, and M. Chertkov, "Options for control of reactive power by distributed photovoltaic generators," Proceedings of the IEEE, vol. 99, no. 6, pp. 1063-1073, 2011.

[5] IEEE PES Industry Technical Support Leadership Committee, "Impact of ieee 1547 standard on smart inverters and the applications in power systems," Technical Report, Aug 2020.
[6] X. Zhou and L. Chen, "An incremental local algorithm for better voltage control in distribution networks," in 2016 IEEE 55th Conference on Decision and Control (CDC), 2016, pp. 2396-2402.

[7] N. Li, G. Qu, and M. Dahleh, "Real-time decentralized voltage control in distribution networks," in 2014 52nd Annual Allerton Conference on Communication, Control, and Computing (Allerton), 2014, pp. 582-588.

[8] P. Jahangiri and D. C. Aliprantis, "Distributed volt/var control by pv inverters," IEEE Transactions on Power Systems, vol. 28, no. 3, pp. 3429-3439, 2013.

[9] F. U. Nazir, B. C. Pal, and R. A. Jabr, "Distributed solution of stochastic volt/var control in radial networks," IEEE Transactions on Smart Grid, vol. 11 , no. 6, pp. 5314-5324, 2020.

[10] _ "A two-stage chance constrained volt/var control scheme for active distribution networks with nodal power uncertainties," IEEE Transactions on Power Systems, vol. 34, no. 1, pp. 314-325, 2019.

[11] K. S. Ayyagari, N. Gatsis, and A. F. Taha, "Chance constrained optimization of distributed energy resources via affine policies," in 2017 IEEE Global Conference on Signal and Information Processing (GlobalSIP), 2017, pp. 1050-1054.

[12] R. A. Jabr, "Linear decision rules for control of reactive power by distributed photovoltaic generators," IEEE Transactions on Power Systems, vol. 33, no. 2, pp. 2165-2174, 2018.

[13] A. Samadi, R. Eriksson, L. Söder, B. G. Rawn, and J. C. Boemer, "Coordinated active power-dependent voltage regulation in distribution grids with pv systems," IEEE Transactions on Power Delivery, vol. 29, no. 3, pp. 1454-1464, 2014.

[14] V. Guigues, "Robust production management," Optimization and Engineering, vol. 10, no. 4, pp. 505-532, 2009.

[15] P. Li, C. Zhang, Z. Wu, Y. Xu, M. Hu, and Z. Dong, "Distributed adaptive robust voltage/var control with network partition in active distribution networks," IEEE Transactions on Smart Grid, vol. 11, no. 3, pp. 2245-2256, 2019.

[16] P. Lagonotte, J. Sabonnadiere, J.-Y. Leost, and J.-P. Paul, "Structural analysis of the electrical system: application to secondary voltage control in france," IEEE Transactions on Power Systems, vol. 4, no. 2, pp. 479486, 1989.

[17] A. Maknouninejad, Z. Qu, J. Enslin, and N. Kutkut, "Clustering and cooperative control of distributed generators for maintaining microgrid unified voltage profile and complex power control," in PES T\&D 2012. IEEE, 2012, pp. 1-8.

[18] B. Zhao, Z. Xu, C. Xu, C. Wang, and F. Lin, "Network partitionbased zonal voltage control for distribution networks with distributed pv systems," IEEE Transactions on Smart Grid, vol. 9, no. 5, pp. 40874098, 2017.

[19] M. Biserica, G. Foggia, E. Chanzy, and J. Passelergue, "Network partition for coordinated control in active distribution networks," in 2013 IEEE Grenoble Conference. IEEE, 2013, pp. 1-5.

[20] Y. Kenan, "A network partition method for power system reactive power control based on power flow tracing," Automation of electric power systems, 2013.

[21] H. Shahbazi and F. Karbalaei, "Decentralized voltage control of power systems using multi-agent systems," Journal of Modern Power Systems and Clean Energy, vol. 8, no. 2, pp. 249-259, 2020.

[22] S. Boyd, N. Parikh, and E. Chu, Distributed optimization and statistical learning via the alternating direction method of multipliers. Now Publishers Inc, 2011.

[23] D. Han and X. Yuan, "A note on the alternating direction method of multipliers," Journal of Optimization Theory and Applications, vol. 155, no. 1, pp. 227-238, 2012.

[24] F. U. Nazir and B. C. Pal, "ADMM based distributed load flow for radial power networks," in 2020 IEEE International Conference on Power Systems Technology (POWERCON). IEEE, 2020, pp. 1-6.

[25] F. Tamp and P. Ciufo, "A sensitivity analysis toolkit for the simplification of mv distribution network voltage management," IEEE Transactions on Smart Grid, vol. 5, no. 2, pp. 559-568, 2014.

[26] K. Christakou, J.-Y. LeBoudec, M. Paolone, and D.-C. Tomozei, "Efficient computation of sensitivity coefficients of node voltages and line currents in unbalanced radial electrical distribution networks," IEEE Transactions on Smart Grid, vol. 4, no. 2, pp. 741-750, 2013.

[27] M. Baran and F. Wu, "Network reconfiguration in distribution systems for loss reduction and load balancing," IEEE Transactions on Power Delivery, vol. 4, no. 2, pp. 1401-1407, 1989.

[28] H. Gu, X. Chu, and Y. Liu, "Partitioning active distribution networks by using spectral clustering," in 2020 IEEE Sustainable Power and Energy Conference (iSPEC), 2020, pp. 510-515. 
[29] R. J. Sánchez-García, M. Fennelly, S. Norris, N. Wright, G. Niblo, J. Brodzki, and J. W. Bialek, "Hierarchical spectral clustering of power grids," IEEE Transactions on Power Systems, vol. 29, no. 5, pp. 22292237, 2014.

[30] M. Farivar and S. H. Low, "Branch flow model: Relaxations and convexification-part i," IEEE Transactions on Power Systems, vol. 28, no. 3, pp. 2554-2564, 2013.

[31] R. Aghatehrani and A. Golnas, "Reactive power control of photovoltaic systems based on the voltage sensitivity analysis," in 2012 IEEE Power and Energy Society General Meeting. IEEE, 2012, pp. 1-5.

[32] R. A. Jabr, "Adjustable robust opf with renewable energy sources," IEEE Transactions on Power Systems, vol. 28, no. 4, pp. 4742-4751, 2013.

[33] Gurobi Optimization, LLC, "Gurobi Optimizer Reference Manual," 2021. [Online]. Available: https://www.gurobi.com

[34] R. Singh, B. C. Pal, and R. A. Jabr, "Statistical representation of distribution system loads using gaussian mixture model," IEEE Transactions on Power Systems, vol. 25, no. 1, pp. 29-37, 2009.

[35] I. Džafić, R. A. Jabr, E. Halilovic, and B. C. Pal, "A sensitivity approach to model local voltage controllers in distribution networks," IEEE Transactions on Power Systems, vol. 29, no. 3, pp. 1419-1428, 2013. 